\title{
Utilizing eHealth and Telemedicine Technologies to Enhance Access and Quality of Consultations: It's Not What You Say, It's the Way You Say It
}

\author{
Katharine Barnard-Kelly, PhD, CPsychol, AFBPsS $S^{1,2}$
}

\begin{abstract}
Therapeutic inertia and suboptimal adherence to therapy have been cited as key contributors to poor diabetes outcomes. Although many of the factors associated with therapeutic inertia and adherence are often beyond the control of both patients and health care providers, most factors can be addressed through improved patientprovider communications.Use of telemedicine and electronic health (eHealth) technologies has the potential to address these factors, and thus, improve management of diabetes, increase access to care, and diminish inequalities in providing health care services. This article examines the behavioral aspects of the eHealth and telemedicine technologies, discusses how patient-centered care can be delivered effectively using eHealth and telemedicine, and reviews a novel model of care, KALMOD, which addresses a range of factors that are critical to improving patient-provider communications and collaborations.
\end{abstract}

Keywords: eHealth, Type 1 diabetes, Type 2 diabetes, Adherence, Inertia, Engagement, Counseling.

\section{Introduction}

$\mathbf{T}$ he International Diabetes Federation (IDF) estimates that global health care spending on diabetes more than tripled to $\$ 548$ billion from 2003 to 2013 , driven by both the increasing number of people with diabetes and increasing spend per capita. ${ }^{1}$ This trend is expected to continue with a further $8 \%$ increase between 2015 and 2017, and is conservatively projected to grow further by 2045 , reaching $\$ 958$ billion. ${ }^{2}$ Moreover, there is significant economic variability in diabetes care provision globally, ${ }^{2}$ which suggests alarming disparities in how countries are addressing the diabetes epidemic and, importantly, the quality of care provided to their diverse populations.

The effectiveness of our spending on diabetes care also raises serious concerns about the future. Despite comprehensive treatment recommendations and the continuing introduction of new diabetes medicines and medical device technologies, a significant percentage of individuals with diabetes are not achieving their treatment goals, resulting in suboptimal clinical outcomes. ${ }^{3,4}$ As reported by the American Diabetes Association (ADA), the direct cost of diabetes in the United States in 2017 was \$237 billion, 62\% (\$157 billion) of which was spent on hospitalizations, emergency room visits, and prescriptions for complications. ${ }^{5}$

Committing large percentages of our diabetes resources to treat the complications of diabetes, rather than preventing complications from occurring through diabetes-prevention programs and improved health care, is both financially unsustainable and untenable as a strategy moving forward.

At a time of rapidly increasing global prevalence of diabetes, exacerbated by increasingly constrained health care resources, novel and innovative modes of treatment delivery are required to bridge the gap. This requirement extends beyond simply translating our current ineffective medical model of health care into new modes of delivery; rather, it demands a paradigm shift in the way that chronic conditions are supported by clinicians, health care systems, and payers.

Use of telemedicine and electronic health (eHealth) technologies has the potential to improve management of chronic diseases such as diabetes, increase access to care, and diminish inequalities in providing health care services. ${ }^{6}$ This article examines the behavioral aspects of these technologies, discusses how patient-centered care can be delivered effectively using eHealth and telemedicine, and reviews a model of care, KALMOD, which addresses a range of factors that

\footnotetext{
${ }^{1}$ Bournemouth University, Bournemouth, United Kingdom.

${ }^{2}$ BHR Ltd., Portsmouth, United Kingdom.
} 
are critical to improving patient-provider communications and collaborations.

\section{Behaviors That Impact Diabetes Outcomes}

Therapeutic inertia and suboptimal adherence to therapy have been cited as key contributors to poor diabetes outcomes. $^{7}$ Therapeutic inertia refers to the failure of health care providers to initiate or intensify therapy in a timely manner according to evidence-based clinical guidelines ${ }^{4,8}$; whereas therapy adherence is defined as the degree to which an individual's behavior corresponds with the agreed recommendations from a health care provider. ${ }^{9}$ Although these terms are useful in identifying the general behaviors associated that can impact clinical outcomes, they do not shed light on the numerous underlying factors and conditions that are driving these behaviors.

It is well known that affordability, complexity of treatment regimen, medication side effects, poor health literacy, and others can all play a major role in patients' ability/willingness to follow their treatment regimens. ${ }^{10}$ Importantly, the impact of psychological factors cannot be ignored. Diabetes distress affects a fifth of people with diabetes,${ }^{11}$ and rates of depression remain high-20\% ${ }^{12}$ to up to $46 \%,{ }^{13}$ depending on the populations studied.

Conversely, although it is assumed that the vast majority of health care providers are dedicated to providing the highest quality of care possible, lack of time, lack of sufficient patient data, and inadequate knowledge contribute significantly to therapeutic inertia. Moreover, inadequate quality metrics and reimbursement models can disincentivize clinicians to devote time/resources for chronic care and create additional time constraints caused by onerous administrative burdens on clinicians and staff. A recent study by Koinis et al. found that workplace stress is an influencer on health care professionals' physical and emotional well-being, curbing their efficiency and negatively impacting their quality of life. ${ }^{14}$

Some of these factors are often beyond the control of both patients and health care providers; however, most can be addressed through improved patient-provider communications. Unfortunately, there exist considerable misunderstandings between patients and their providers.

Results from two recent surveys of patients with type 2 diabetes $(n=1000)$ and health care providers $(n=1004)$ conducted by the American Association of Clinical Endocrinologists in partnership with Sanofi showed notable discordance between patients' beliefs and health care providers' perceptions of patient beliefs. ${ }^{15}$ Although 52\% of patients reported that they would be very willing to make multiple medication changes, only $19 \%$ of clinicians thought that their patients would be willing to make these changes. The surveys also found that the longer patients go without achieving their individualized $\mathrm{HbA1c}$ targets, the greater their frustration. Importantly, 22\% of patients surveyed reported that they stopped taking their medications without telling their health care provider, and among those patients, $38 \%$ reported discontinuing their medication due to frustration in not meeting the HbA1c targets. These findings clearly illustrate that most patients are willing to take action with their diabetes management but are frustrated by their inability to achieve their diabetes goals.

What about those patients who are adhering to their prescribed therapy? Although much attention has been focused on developing interventions to improve medication adherence, an earlier study of $>11,000$ insured patients with diabetes showed that $>50 \%$ could not achieve their glycemic targets despite their adherence to therapy. ${ }^{16}$

These findings do not fit with the argument that patients are not trying hard enough to manage their diabetes. However, they do suggest that health care professionals may not understand or appreciate the demands of diabetes from a lived experience perspective - the relentless daily demands made of people with diabetes worldwide. It is clear that health care professionals have a desire to motivate their patients; however, they often lack the skills to do so. Unfortunately, current approaches to patient-provider communications are inadequate.

At present, consultations are designed to focus on biomedical outcomes by using a didactic medical model. Guidelines dictate use of complex and detailed algorithms for the management of blood glucose, lipids, blood pressure, and long-term complications; however, they do not address patients' psychosocial needs or outcomes. Although patientprovider communication remains a cornerstone of health care, evidence shows that major problems exist within consultations, resulting in poorer outcomes and dissatisfaction for patients and health care professionals.

An earlier study by Beckman and Frankel found that physicians typically interrupt their patients $18 \mathrm{~s}$ after they start describing their problems. ${ }^{17}$ During the 74 clinician visits studied, the patient was provided the opportunity to complete his or her opening statement of concerns in only 17 (23\%) of the visits. A later study showed that half of patients' concerns are not discussed at all, and that in $\sim 50 \%$ of consultations, patients and physicians disagree entirely on the central problem presented. ${ }^{18}$ As noted by DiMatteo, insufficient explanation of therapy goals and strategies often results in poor patient understanding, and lack of consensus between doctor and patient can lead to therapeutic failure and poor clinical outcomes. ${ }^{19}$

\section{Current Communication Theories}

A number of communication theories exist to guide interactions between health care professionals and their patients, as well as between health care professions.

\section{Dyadic interpersonal communication model}

One theory is the dyadic interpersonal communication model, which is most commonly used in medical consultations. ${ }^{20}$ This approach describes the dynamic interactive process between two people. It is based on a sender and recipient model, with outside influences (e.g., perception, attitude, content, emotional, and physical components) that can impact the interaction. The recipient (e.g., person with diabetes) is responsible for decoding the information given by the sender (e.g., health care professional).

However, there is an inherent power imbalance in routine consultations between health care providers and their patients. ${ }^{21}$ Physicians hold status and authority through the cultural and symbolic aspects of medicine. Although they exert this power during consultations, they have little conscious awareness that this is happening. ${ }^{21}$ Because patients are vulnerable due to their need for health care, this paternalistic approach to patient-provider relationships immediately creates a power imbalance; patients become recipients 
of health care rather than partners in their disease management. This relationship is incongruent with the role patients must play in real life. It has been estimated that people with diabetes must manage their diabetes alone for $95 \%$ of the time, ${ }^{22}$ and so must become expert in the required selfmanagement tasks and find ways to cope with the demands of the disease as well as its management.

\section{Peplau's Interpersonal Relations Theory}

Another theory is Peplau's Interpersonal Relations Theory, which focuses on the therapeutic process taking place. ${ }^{23}$ The theory defines four stages:

- Orientation Phase: health care professionals engage the patient in treatment, and the patient is able to ask questions and receive explanations and information. This stage is designed to help the patient develop trust and is where first impressions about the health care professional and health care system begin to evolve.

- Identification Phase: The patient and nurse begin to work together. These interactions provide the basis for understanding, trust, and acceptance as the patient becomes an active participant in treatment.

- Exploitation Phase: The patient takes advantage of all services offered, exploiting the nurse-patient relationship to address treatment goals.

- Resolution Phase: As a result of effective communication, the patient's needs are met, and he or she moves toward full independence. The patient no longer needs help, and the relationship ends.

There are several limitations to this approach in supporting people with chronic conditions such as diabetes. First, the orientation phase focuses on the provision of information and explanations but fails to recognize the value of information provided by the person with diabetes. Second, the identification phase recognizes the person with diabetes as simply an active participant in treatment, rather than an equal member of the health care team who brings considerable skill and expertise. Third, the exploitation phase situates the person with diabetes as a passive recipient of health care rather than having autonomy over his/her own needs. Finally, by necessity, the relationship never ends. Diabetes is a chronic lifelong illness with no cure, no quick fix, and no one-time solution. As diabetes progresses, therapy changes are often needed, adding additional complexity and burden, which can add to the patients' ability to manage their diabetes and comorbidities as well as negatively impact quality of life.

\section{Kaleidoscope model of care}

The kaleidoscope model is a dynamic adaptive approach to identifying and addressing individuals' needs at their clinical encounters and throughout each stage of their life with diabetes. $^{24}$ Importantly, the person with diabetes is at the heart of decision-making with the availability of therapy, education, and support tailored to provide individualized care. What is novel is that it supports health care professionals in providing care pathways that are mapped to individual's priorities.

The model incorporates existing regimen factors that address primarily the clinical aspects of diabetes care and selfmanagement (e.g., medications, devices, and education), and pulls these together into a holistic wide-reaching model that includes both external factors (social support, resources, and environment) and internal factors (intrinsic feelings, beliefs, and motivations) to address all aspects of living with diabetes. These factors are separated into distinct but linked core components, or "cogs" that interact to reflect individuals' experience and priorities for their specific treatment needs (Fig. 1).

The cogs represent internal and external factors affecting behavior and form the basis for an individualized diabetes care plan. Thus, as individual priorities for each component are assessed and prioritized, available care pathways are mapped to each priority to provide choice and become the focus of clinical discussion. For example, "social support," "beliefs," and "education" may take priority at one time; however, this may change to "environment," "feelings," and "education" as life circumstances change. Thus, the level of social support and education that an individual requires on diagnosis may subside as motivation and feelings take priority over time. Changes to therapy or device choices (e.g., self-monitoring of blood glucose (SMBG)) may bring education back to the fore and highlight resource needs and health beliefs.

\section{Role of Telemedicine/eHealth Technologies}

Remote consultations and data sharing between health care professionals and their patients through video conferences, telephone, texting, and other eHealth technologies offer new opportunities to interact and engage with large number of patients over distance in innovative and cost-effective ways. Studies have shown that use of telemedicine strategies to share glucose data, monitor patient health status, and adjust therapy has been shown to improve glycemic control, ${ }^{25-29}$ patient self-efficacy, ${ }^{30,31}$ treatment satisfaction, ${ }^{6,29}$ and quality of life $\mathrm{e}^{25,29}$ in individuals with diabetes.

Increasing adoption of these technologies appears to be driven by a number of factors:

- Reduced patient burden. The ability to share data and receive advice from home or other remote locations reduces the time and inconvenience of travel to clinic visits.

- Improved chronic care delivery. Use of these technologies has the potential to shift the paradigm from acute care to more effective chronic disease management of larger number of patients at lower per-patient costs. ${ }^{32}$

- Potential to address therapeutic inertia. It is well known that lack of patient data contributes to inadequate intensification of diabetes therapy, which can lead to suboptimal glycemic control. ${ }^{7,33,34}$ As demonstrated by Mora et al., the availability of reliable near real-time glucose data, transmitted automatically to clinicians in structured formats, increased the number and frequency of therapy changes in individuals with insulin-treated diabetes.

- Increased access to telemedicine/eHealth technologies. There are currently 2.7 billion active smartphone users worldwide. ${ }^{35}$ As use of smartphone technology continues to accelerate, the number of individuals with access to telemedicine and eHealth technologies will continue to increase.

Despite the demonstrated and potential benefits of using telemedicine/eHealth, it is important to remember that these 


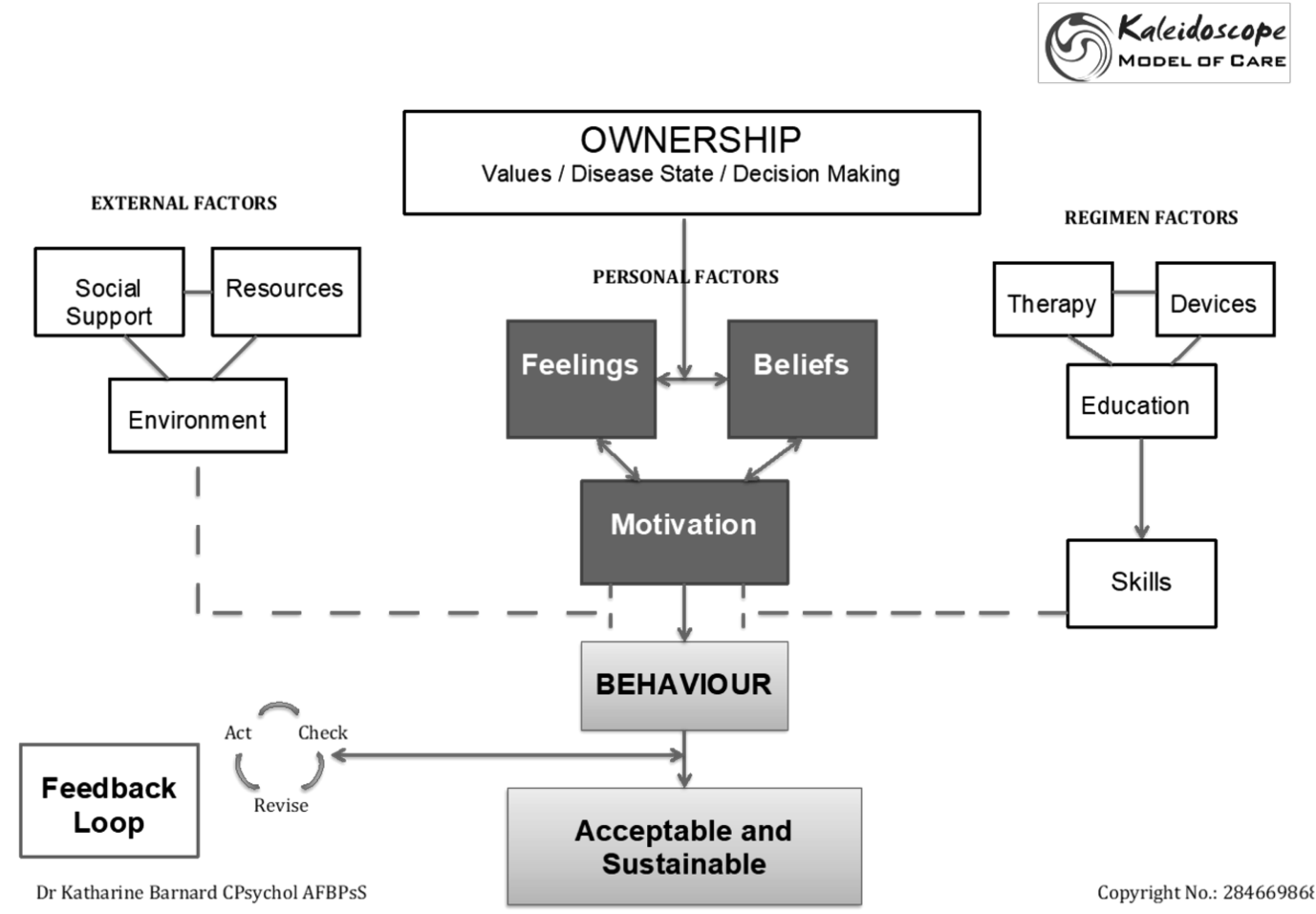

FIG. 1. Kaleidoscope model. Figure shows the distinct but interconnecting aspects relevant to diabetes management. The health beliefs and values of the individual drive understanding and subsequent actions. Self-management behavior is influenced by each aspect to some extent at any given time, it is dynamic and responsive to changes in those facilitators and barriers over time. All actions must be acceptable, achievable, and sustainable, and as such open to review between physicians and the individual over time.

technologies are only tools. Simply moving from one delivery method to another does not address the content or quality of interactions between health care providers and their patients. The content of those touch points and consultations must ensure that the same problems encountered in face-toface consultations are not simply delivered electronically. As discussed, routine face-to-face consultations in specialist and primary care settings often leave both patients and health care professionals feeling frustrated. Telemedicine consultations do not alter this fact. Rather, they may actually create additional problems if they are not structured to address the full range of patient needs and concerns.

As recommended in national and international guidelines, patient-provider interactions must address both the psychological and biomedical needs. The National Institute for Health and Care Excellence recommends that all interactions should be "needs-led," and the psychological needs of people with diabetes should be addressed in an organized and planned way. ${ }^{36,37}$ Other national and international guidelines provide similar recommendations. For example, the IDF states that "healthcare professionals should, in communicating with a person with diabetes, adopt a whole-person approach and respect the person's central role in their ongoing diabetes education and care.",38 The American Diabetes Association guidelines recommend that assessment of psychological and social situations should be included as an on-going part of the medical management of diabetes. ${ }^{39}$ Yet, provision of services for psychological support remains stubbornly absent from routine clinical care.

\section{KALMOD}

KALMOD is a novel clinical tool based on the kaleidoscope model of care. ${ }^{24}$ The tool utilizes a decision-tree questionnaire that is designed to enhance patient-provider consultations by identifying patient priorities and facilitating the development of treatment strategies that address the individual holistic needs of each patient. The goal is to facilitate more collaborative patient-centered relationships between people with diabetes and their health care providers by identifying and articulating barriers to optimal diabetes control.

The first step in the KALMOD process is to assess the patients' status, documenting their personal history (e.g., socioeconomic situation and available resources) and medical history. A brief questionnaire was administered to obtain information about patients' specific priorities, according to their health beliefs, level of motivation, self-efficacy, and feeling of empowerment.

Responses are analyzed using an algorithm, and a pictorial representation of current needs is presented, along with the identification of potential discussion topics, priorities for behavior change, or therapy modification. The one-page summary provides an easy-to-read visual snapshot of current diabetes needs, priorities, and care pathway options for discussion. Based on this discussion, patients and their health care providers can then formulate a mutually agreed upon action plan for moving forward.

The KALMOD assessment is web based and easily accessible on a number of devices, including smartphones, 
tablets, and computers. As such, the questionnaire can be completed in the waiting room at clinic or at home before consultations, with the results available for discussion at the clinic visit. Printouts can be made available for patients to take home, with copies placed on file at clinic.

Clinical trials to assess the utility and feasibility of the KALMOD tool are currently underway; however, preliminary data from pilot studies show that KALMOD is acceptable, relevant, and tailored to individual needs, and that it can be implemented in clinical practices. ${ }^{40}$ These analyses were conducted in two phases. The first phase focused only on people with diabetes and whether the tool met their needs in terms of acceptability, usability, and relevance to their own specific needs and priorities. After this, minor modifications were made to wording of questions and refinement of weightings applied. The second phase was conducted in December 2018 and January 2019 with people with diabetes and health care professionals. These analyses focused on acceptability of the revised tool for people with diabetes, as well as usefulness and relevance within routine clinical care for health care professionals.

In the first phase analyses, three focus groups $(n=12)$ and six interviews were conducted with participants completing the tool and providing feedback, with a further 13 participants completing the tool and providing feedback through an online survey. Key positive themes were ease of use, relevance, personalized feedback, and simplicity of the tool, for example, "I love this, quick simple and effective." The tool was revised in line with recommendations regarding wording of some questions to aid clarity and remove ambiguity. Survey data showed that all participants believed the tool was intuitive and simple to use, relevant, and easy to understand. Sixty-nine percent $(n=9)$ thought it would improve their consultation with their health care team and $61 \%(n=8)$ felt it would help the health care team better understand their needs.

The second phase analyses focused on acceptability of the revised tool for people with diabetes, as well as usefulness and relevance within routine clinical care for health care professionals. One-to-one interviews were conducted with 12 people with diabetes ( 7 female, 5 male aged $18-74$ years) and 8 health care professionals ( 4 diabetologists, 2 specialist, and 2 applied health professionals). All participants expressed enthusiasm for the tool and reported that it accurately identified personal priorities and clear appropriate care pathways to meet those needs. All health care professionals stated that it would be useful in clinic to potentially reduce consultation times and improve communication, as well as aiding greater understanding of patient needs. Minor further revisions were recommended to question structure. A clinical trial is in planning and due to commence in mid-2019.

\section{Conclusions}

The burgeoning global diabetes epidemic is threatening the health and well-being of millions. The growing costs of diabetes, particularly those associated with the management of complications, continue to stress our already burdened health care systems. Diabetes is a chronic disease, and transition from current acute care models of care to more appropriate chronic care models is needed.

As individuals adapt to life with diabetes, their therapy, support, and education provision also must adapt to fill specific knowledge and support gaps. Structured systematic assess- ment of patient needs and barriers can guide and focus consultations in ways that enable health care providers support their patients with individualized holistic care. To achieve this, it is necessary to look beyond current approaches.

Among the evolving eHealth offerings, new decision support tools are being designed to aid medical decisions and improve understanding between the person with diabetes and the health care team. Effective use of telemedicine and eHealth technologies has the potential to affect this paradigm shift. As large technology companies (e.g., Google, Amazon, and Sony) move into the health care space, we will continue to see a growing emphasis on digitization of health care solutions. Whether these solutions result in improved biomedical and psychosocial outcomes for people with diabetes, and whether those improvements can be sustained in the long term, remains to be seen.

What is clear, however, is that the face of health care is changing, and the person with diabetes must be considered an equal partner rather than a recipient of such innovations. Only then will it be possible to ensure that change is positive. Although technologies come and go, conversation persists, and the content, style, and usefulness of those conversations remain crucially important irrespective of the way they are delivered; consultations remain a very human experience.

The KALMOD tool enables health care providers to easily identify individual needs and provide appropriate feasible choices for people with diabetes. This, in turn, has the potential to facilitate more informed decision-making and personalization of education, therapies, devices, and support tailored to that individual.

We believe the KALMOD approach can streamline health care delivery during consultations and remove the pressure from health care professionals to provide "the answer" without having the required information about patients' individual needs and barriers to effective self-management. Moreover, because KALMOD matches patients with treatment options/devices that are sustainable in the long term, the approach has the potential to create greater economic efficiencies for health care systems by improving therapy adherence and minimizes discontinuation of medical device use.

\section{Acknowledgments}

The author wishes to thank Christopher G. Parkin (CGParkin Communications, Inc., Henderson, NV) for editorial assistance in developing this article. Roche Diabetes Care provided funding for the development of this article.

\section{Author Disclosure Statement}

K.B. has received honoraria for participation in the Roche Global Advisory Board and Insulin Delivery System Advisory Board, Sanofi Integrated Care Advisory Board. She has received research funding from Silvercloud Health, IDEO, Senseonics, and Novo Nordisk, and has received honoraria from Lifescan and Sanofi.

\section{References}

1. International Diabetes Federation: IDF Diabetes Atlas, $6^{\text {th }}$ ed. Brussels: International Diabetes Federation, 2013. www .idf.org/e-library/epidemiology-research/diabetes-atlas/19atlas-6th-edition.html (accessed December 3, 2018). 
2. International Diabetes Federation: IDF Diabetes Atlas, $8^{\text {th }}$ ed. Brussels: International Diabetes Federation, 2018. www .idf.org/e-library/epidemiology-research/diabetes-atlas/134idf-diabetes-atlas-8th-edition.html (accessed December 3, 2018).

3. Carls G, Huynh J, Tuttle E, et al.: Achievement of glycated hemoglobin goals in the US remains unchanged through 2014. Diabetes Ther 2017;8:863-873.

4. Khunti K, Gomes MB, Pocock S, et al.: Therapeutic inertia in the treatment of hyperglycaemia in patients with type 2 diabetes: a systematic review. Diabetes Obes Metab 2018; 20:427-437.

5. American Diabetes Association: Economic costs of diabetes in the U.S. in 2017. Diabetes Care 2018;41:917-928.

6. Mair F, Whitten P: Systematic review of studies of patient satisfaction with telemedicine. BMJ 2000;320:1517-1520.

7. Khunti K, Wolden ML, Thorsted BL, et al.: Clinical inertia in people with type 2 diabetes: a retrospective cohort study of more than 80,000 people. Diabetes Care 2013;36:34113417.

8. Safford MM, Shewchuk R, Qu H, et al.: Reasons for not intensifying medications: differentiating "clinical inertia" from appropriate care. J Gen Intern Med 2007;22:1648-1655.

9. World Health Organization: 2003. Adherence to long term therapies: evidence for action. www.who.int/chronic conditions/en/adherence_report.pdf (accessed December 3, 2018).

10. Ross SA: Breaking down patient and physician barriers to optimize glycemic conrol in type 2 diabetes. Am J Med 2013;126(9 Suppl 1):S38-S48.

11. Gahlan D, Rajput R, Gehlawat P, Gupta R: Prevalence and determinants of diabetes distress in patients of diabetes mellitus in a tertiary care centre. Diabetes Metab Syndr 2018;12:333-336.

12. Salinero-Fort MA, Gómez-Campelo P, San Andrés-Rebollo FJ, et al.: Prevalence of depression in patients with type 2 diabetes mellitus in Spain (the DIADEMA Study): results from the MADIABETES cohort. BMJ Open 2018. http://dx .doi.org/10.1136/bmjopen-2017-020768. Accessed December 4, 2018.

13. Das R, Singh O,Thakurta RG, et al.: Prevalence of depression in patients with type II diabetes mellitus and its impact on quality of life. Indian J Psychol Med 2013;35: 284-289.

14. Koinis A, Giannou V, Drantaki V, et al.: The impact of healthcare workers job environment on their mentalemotional health. Coping strategies: the case of a local general hospital. Health Psychol Res 2015;3:1984.

15. Fonseca VA, Grunberger G, Anhalt H, et al. for the Consensus Conference Writing Committee: Continuous glucose monitoring: a consensus conference of the American Association of Clinical Endocrinologists and American College of Endocrinology. Endocr Pract 2016;22:1008-1021.

16. Juarez DT, Ma C, Kumasaka A, et al.: Failure to reach target glycated A1c levels among patients with diabetes who are adherent to their antidiabetic medication. Popul Health Manag 2014;17:218-223.

17. Beckman HB, Frankel RM: The effect of physician behavior on the collection of data. Ann Intern Med 1984;101: 692-696.

18. Barnard KD, Cradock S, Parkin T, Skinner TC: Effectiveness of a computerised assessment tool to prompt individuals with diabetes to be more active in consultations. Pract Diabetes Int 2006;23:36-41.
19. DiMatteo MR: The role of the physician in the emerging health care environment. West J Med 1998;168:328-333.

20. Berlo DK: The Process of Communication: An Introduction to Theory and Practice. New York: Holt, Rinehart, Winston, 1960.

21. Nimmon L, Stenfors-Hayes T: The 'handling' of power in the physicial-patient encounter: perceptions from experienced physicians. BMC Med Educ 2016;16:114.

22. Funnell MM, Anderson RM: The problem with compliance in diabetes. JAMA 2000;13:1709.

23. Peplau HE: Interpersonal Relations in Nursing. New York: G. P. Putnam's Sons, 1952.

24. Barnard KD, Lloyd CD, Dyson PA, et al.: Kaleidoscope model of diabetes care: time for a rethink? Diabet Med 2014;31:522-530.

25. Bujnowska-Fedak MM, Puchala E, Steciwko A: The impact of telehome care on health status and quality of life among patients with diabetes in a primary care setting in Poland. Telemed J E Health 2011;17:153-163.

26. Charpentier G, Benhamou PY, Dardari D, et al.: The Diabeo software enabling individualized insulin dose adjustments combined with telemedicine support improves HbAlc in poorly controlled type 1 diabetic patients: a 6-month, randomized, open-label, parallel-group, multicenter trial (TeleDiab 1 Study). Diabetes Care 2011;34:533-539.

27. Stone RA, Rao RH, Sevick MA, et al.: Active care management supported by home telemonitoring in veterans with type 2 diabetes: the DiaTel randomized controlled trial. Diabetes Care 2010;33:478-484.

28. Wakefield BJ, Holman JE, Ray A, et al.: Effectiveness of home telehealth in comorbid diabetes and hypertension: a randomized, controlled trial. Telemed J E Health 2011;17: 254-261.

29. Mora P, Buskirk A, Lyden M, et al.: Use of a novel, remotely connected diabetes management system is associated with increased treatment satisfaction, reduced diabetes distress, and improved glycemic control in individuals with insulin-treated diabetes: first results from the Personal Diabetes Management study. Diabetes Technol Ther 2017;19:715-722.

30. Trief PM, Teresi JA, Eimicke JP, et al.: Improvement in diabetes self-efficacy and glycaemic control using telemedicine in a sample of older, ethnically diverse individuals who have diabetes: the IDEATel project. Age Ageing 2009;38:219-225.

31. Beck RW, Connor CG, Mullen DM, et al.: The fallacy of average: how using HbA1c alone to assess glycemic control can be misleading. Diabetes Care 2017;40:994-999.

32. Warren R, Carlisle K, Mihala G, Scuffham PA: Effects of telemonitoring on glycaemic control and healthcare costs in type 2 diabetes: a randomised controlled trial. J Telemed Telecare 2018;24:586-595.

33. Khunti K, Nikolajsen A, Thorsted BL, et al.: Clinical inertia with regard to intensifying therapy in people with type 2 diabetes treated with basal insulin. Diabetes Obes Metab 2016;18:401-409.

34. Ziemer DC, Miller CD, Rhee MK, et al.: Clinical inertia contributes to poor diabetes control in a primary care setting. Diabetes Educ 2005;31:564-571.

35. Pew Research Center: 10 facts about smartphones as the iPhone turns 10. Mobile Fact Sheet. www.pewresearch.org/ fact-tank/2017/06/28/10-facts-about-smartphones (accessed July 12, 2018).

36. National Institute for Health and Care Excellence (NICE): The management of type 2 diabetes in adults: management. 
NICE guideline (NG28). May 2017. www.nice.org.uk/ guidance/ng28 (accessed December 3, 2018).

37. National Institute for Health and Care Excellence: Type 1 diabetes in adults: diagnosis and management. 2015. www. niceorg.uk/guidance/ng17 (accessed February 2, 2018).

38. International Diabetes Federation: IDF Clinical Guidelines Task Force. Global Guideline for Type 2 Diabetes. Brussels: International Diabetes Federation, 2005. www.idf.org/ e-library/guidelines/79-global-guideline-for-type-2-diabetes (accessed December 3, 2018).

39. Beck J, Greenwood DA, Blanton L, et al.: National standards for diabetes self-management education. Diabetes Care 2017;40:1409-1419.
40. Barnard KD, Hitchcock J, Hood KK, et al.: KALMOD: novel holistic clinical tool to improve consultations and outcomes. Diabetes 2017;66(Suppl 1A):89-LB

Address correspondence to: Katharine Barnard-Kelly, PhD, CPsychol, AFBPsS 42 Kilmiston Drive Fareham, Nr Portsmouth Hants PO16 8EG United Kingdom

E-mail: katharinebarnard@bhrltd.com 